DOI 10.4171/JEMS/275

N. Nikolov $\cdot$ L. Pyber

\title{
Product decompositions of quasirandom groups and a Jordan type theorem
}

Received January 20, 2009 and in revised form October 12, 2009

\begin{abstract}
We first note that a result of Gowers on product-free sets in groups has an unexpected consequence: If $k$ is the minimal degree of a representation of the finite group $G$, then for every subset $B$ of $G$ with $|B|>|G| / k^{1 / 3}$ we have $B^{3}=G$.

We use this to obtain improved versions of recent deep theorems of Helfgott and of Shalev concerning product decompositions of finite simple groups, with much simpler proofs.

On the other hand, we prove a version of Jordan's theorem which implies that if $k \geq 2$, then $G$ has a proper subgroup of index at most $c_{0} k^{2}$ for some constant $c_{0}$, hence a product-free subset of size at least $|G| /(c k)$. This answers a question of Gowers.
\end{abstract}

\section{Introduction}

Sum-free subsets of abelian groups have been much investigated in the past 40 years. Very recently Green and Ruzsa [GR] have determined the maximal size of a sum-free subset of any finite abelian group.

A subset $X$ of a not necessarily abelian group $G$ is called product-free if there are no solutions to $x y=z$ with $x, y, z \in X$. The maximal size $\alpha(G)$ of a product-free subset of a finite group $G$ has been considered by Babai and Sós $[\overline{\mathrm{BS}}]$. For example, they proved that for any soluble group $G$ of order $n$ we have $\alpha(G) \geq 2 n / 7$ and asked whether a similar linear bound holds for arbitrary finite groups.

Note that any nontrivial coset of a subgroup is product-free. In fact, in 1950 (according to [S p. 246]) Moser conjectured that the largest product-free subsets of alternating groups are cosets of maximal subgroups.

Kedlaya [Ke] disproved this, by showing that if a subgroup $H$ has index $m$ in a group $G$, then one can actually find a set of size at least $\mathrm{cm}^{1 / 2}|H|$ that is product-free. Here and below $c$ denotes an absolute positive constant.

Combining this estimate with the classification of finite simple groups (CFSG) Kedlaya showed that for every finite group $G$ we have $\alpha(G) \geq c n^{11 / 14}$. He asked whether for every $\varepsilon>0$ one can obtain a bound of $c(\varepsilon) n^{1-\varepsilon}$.

A negative answer to the above question was obtained very recently by Gowers [Gow]. He showed that for sufficiently large $q$ the group $\Gamma=P S L(2, q)$ has no product-free

N. Nikolov: Department of Mathematics, Imperial College, London, UK; e-mail: nnikolov@imperial.ac.uk

L. Pyber: A. Rényi Institute of Mathematics, H-1053 Budapest, Hungary; e-mail: pyber@ renyi.hu 
subsets of size $c|\Gamma|^{8 / 9}$. His proof depends on the fact, proved by Frobenius, that every nontrivial representation of $\operatorname{PSL}(2, q)$ has degree at least $(q-1) / 2$.

Gowers went on to consider combinatorial properties of finite groups $G$ such that every nontrivial representation of $G$ has degree at least $k$. He calls such groups quasirandom, since this property turned out to be equivalent to several other properties, some of which state that certain associated graphs are quasirandom (see [Gow] for a detailed discussion of quasirandom graphs).

Gowers proved [Gow, p. 22] the following general result on product-free sets of quasirandom groups.

Proposition 0. Let $G$ be a group of order $n$ such that the minimal degree of a nontrivial representation is $k$. If $A, B, C$ are three subsets of $G$ such that $|A||B||C|>n^{3} / k$, then there is a triple $(a, b, c) \in A \times B \times C$ such that $a b=c$.

The starting point of the present paper is the following surprising consequence.

Corollary 1. Let $G$ be a group of order $n$ such that the minimal degree of a nontrivial representation is $k$. If $A, B, C$ are three subsets of $G$ such that $|A||B||C|>n^{3} / k$, then $A \cdot B \cdot C=G$. In particular, if, say, $|B|>n / k^{1 / 3}$, then $B^{3}=G$.

Proof. Consider the set $G \backslash A B$. By Proposition 0 the size of this set is strictly less than $|C|$, i.e., we have $|A B|+|C|>|G|$. It follows that for any $g \in G$ the intersection of the sets $A B$ and $g C^{-1}$ is nonempty, which implies $g \in A B C$.

Applying Corollary 1 to the sets $A, B, C^{-1}$ we see that Proposition 0 and Corollary 1 are in fact equivalent.

Corollary 1. apart from its intrinsic interest, seems to be an extremely useful tool. Recently a number of deep theorems have been obtained concerning product decompositions of simple groups. Corollary 1 can be used to give short and relatively elementary proofs, while improving the results. It is particularly useful in the case of simple groups of Lie type. For these groups rather strong lower bounds on the minimal degrees of their nontrivial representations are known.

For a (possibly twisted) Lie type $L$ other than ${ }^{2} B_{2},{ }^{2} G_{2},{ }^{2} F_{4}$ define the rank $r=r(L)$ to be the untwisted Lie rank of $L$ (that is, the rank of the ambient simple algebraic group), and for $L$ of type ${ }^{2} B_{2},{ }^{2} G_{2},{ }^{2} F_{4}$ define $r(L)=1,1,2$ respectively.

It follows from [LS] that there is a constant $c$ such that for any simple group $L$ of Lie type of rank $r$ over $\mathbb{F}_{q}$ we have $k \geq c q^{r}$ for the minimal degree $k$ of a nontrivial representation of $L$. For $L=\operatorname{PSL}(n, q)$ we obtain the following.

Proposition 2. Let $B$ be a subset of $L$ of size at least $2|L| / q^{(n-1) / 3}$. Then $B^{3}=L$.

A similar result in the case of $\Gamma=P S L(2, p), p$ prime, plays an important role in the proof of a recent breakthrough result of Helfgott concerning the diameter of Cayley graphs of $\Gamma$.

Helfgott [He1] showed that for every set of generators $X$ of $\Gamma$ every element of $\Gamma$ can be expressed as a product of at most $O\left((\log p)^{c}\right)$ elements of $X \cup X^{-1}$. 
Proposition 2 improves (the easier part of) his Key Proposition [He1, p. 2] even for $\Gamma=P S L(2, p)$ and implies that in fact every element of $\Gamma$ can be expressed as a product of at most $O\left((\log p)^{c}\right)$ elements of $X$ itself (this improvement also follows from the results in $[\overline{\mathrm{Ba}}])$.

It is an open problem whether Helfgott's result extends to $\operatorname{PSL}(n, p)$. Proposition 2 may be useful in obtaining a positive answer. (It has indeed been used very recently by Helfgott in [He2] where he obtains an $O\left((\log p)^{c}\right)$ bound for the diameters of Cayley graphs of $\operatorname{PSL}(3, p)$.)

As another interesting application we prove the following Waring type theorem. For a group word $w=w\left(x_{1}, \ldots, x_{d}\right)$ let $w(G)$ denote the set of values of $w$ in $G$.

Theorem 3. Let $k \geq 1$ and $\bar{w}=\left\{w_{1}, \ldots, w_{k}\right\}$ be a set of nontrivial group words. Let $L$ be a finite simple group of Lie type of rank $r$ over the field $F_{q}$ and set $\bar{w}(L)=w_{1}(L) \cap$ $\cdots \cap w_{k}(L)$. Let $W$ be any subset of $\bar{w}(L)$ such that $|W| \geq|\bar{w}(L)| / q^{r / 13}$. There exists a positive integer $N$ depending only on $\bar{w}$ such that if $|L|>N$, then

$$
W^{3}=L .
$$

As the main result of a difficult paper Shalev [Sh1] has obtained the same result in the case $k=1$ and $W=\bar{w}(L)$ (allowing $L$ to be also an alternating group) 1

Combining the methods of that paper with [LSh1] one can prove it for $W=\bar{w}(L)$ and $k$ arbitrary. An advantage of our sparse version is that one can impose further restrictions on $W$. For example one can require that no two elements of $W$ are inverses of each other or images of each other under Frobenius automorphisms. In addition, (using the first part of Corollary (1) it follows that every element $g \in L$ is a product $g=h_{1} h_{2} h_{3}$ of distinct $h_{i} \in \bar{w}(L)$. It will be interesting to see if $h_{i}$ can be taken to be pairwise noncommuting elements from $\bar{w}(L)$, or such that $\left\langle h_{1}, h_{2}, h_{3}\right\rangle=G$; this does not seem to follow immediately from Corollary 1 .

Shalev's proof in [Sh1] relies on a whole array of deep results on the character theory of simple groups, developed to estimate the diameters of Cayley graphs of simple groups with respect to conjugacy classes.

Our proof of Theorem 3 is relatively short compared with [Sh1] and uses an auxiliary result from [LSh1] (see Proposition 1.2 below). This says roughly that for simple groups of Lie type not of type $A_{r}$ or ${ }^{2} A_{r}$ the sets $\bar{w}(L)$ are "very large".

For groups of type $A_{r}$ and ${ }^{2} A_{r}$ we provide somewhat weaker estimates for $|\bar{w}(L)|$ which still make Corollary 1 applicable. It would be most useful to obtain analogues of Corollary 1 for smaller sets $B$. The following results indicate how far one can go in this direction.

Theorem 4. Let $G$ be a finite linear group of degree $k$ over the complex field. Then $G$ has a permutation representation of degree at most $c_{0} k^{2}$ with abelian kernel, where $c_{0}<10^{10}$ is an absolute constant.

\footnotetext{
1 We remark that for alternating groups and simple groups of Lie type of bounded rank it was shown later in [LSh1] that (for arbitrary $k$ ) in fact $\bar{w}(L)^{2}=L$ if $L$ is large enough.
} 
The proof of this result relies on CFSG. As an immediate consequence we obtain the following.

Corollary 5. Let $G$ be a finite group such that $G$ has an irreducible representation of degree $k \geq 2$. Then $G$ has a proper subgroup $H$ of index at most $c_{0} k^{2}$.

In particular $H$ is a subset of size at least $n /\left(c_{0} k^{2}\right)$ in $G$ which does not even generate $G$.

As a "partial converse" to Proposition 0 Gowers [Gow proved that if a group $G$ contains no large product-free subsets, then it is quasirandom. More precisely, he gave an elementary argument showing that if the minimal degree of a representation of $G$ is $k$, then $G$ has a product-free subset of size at least $n / c^{k}$ for some absolute constant $c>1$. Gowers asked whether this can be improved to $n / k^{c}$ (for $k \geq 2$ ). Applying Kedlaya's result to $H$ as above we see that $G$ has a product-free subset of size at least $n /(c k)$ for some constant $c$, i.e. we obtain a positive answer to his question.

Finally, for completeness in the last section we present a simplified version of Gowers' proof of Proposition 0 in the special case when one of the sets $A, B$ or $C$ is symmetric. This case is enough for most of our applications above.

\section{Waring type theorems}

The main result of [Sh1] is the following

Theorem 1.1. Let $w \neq 1$ be a group word. Then there exists a positive integer $N=$ $N(w)$ such that for every nonabelian finite simple group $G$ with $|G| \geq N$ we have

$$
w(G)^{3}=G .
$$

For example, each $g \in G$ can be expressed as a product of three $k$-th powers.

The proof in [Sh1] relies on algebraic geometry via [La], the Deligne-Lusztig theory of characters of Chevalley groups and on a recent work on character-theoretic zeta functions [LSh2].

In this section we indicate a proof of Theorem 3 . It is a generalization of the above theorem for groups of Lie type which does not use such difficult character-theoretic tools. We rely instead on some very recent results of Larsen and Shalev. In [LSh1] they give a short proof of the following.

Proposition 1.2. Let $k \geq 1$ and $\bar{w}=\left\{w_{1}, \ldots, w_{k}\right\}$ be a set of words. Let L be a finite simple group of Lie type of rank $r$, which is not of type $A_{r}$ or ${ }^{2} A_{r}$. There is an absolute constant $c$ and an integer $N=N(\bar{w})$ such that if $|L| \geq N$ then

$$
|\bar{w}(L)| \geq c|L| / r .
$$

The proof of Proposition 1.2 relies on algebraic geometry (replacing [La]) and grouptheoretic arguments. It also applies to groups of type $A_{r}$ and ${ }^{2} A_{r}$ for $r$ bounded and in fact to their covering groups $S L(r+1, q), S U(r+1, q)$. 
As noted in the introduction, if $L$ is a simple group of Lie type over $\mathbb{F}_{q}$, then we have $k \geq c q^{r}$ for the minimal degree $k$ of a representation of $L$. Hence Proposition 1.2 and Corollary 1 immediately imply Theorem 3 if $L$ is not of type $A_{r}$ or ${ }^{2} A_{r}$ with $r \geq 1000$, say.

For the groups $S L(n, q)$ and $S U(n, q)$ the minimal degree of a representation is at least $\left(q^{n-1}-1\right) / 2$ (see [LS]). To complete the proof of Theorem 3 we have to show that for these groups we have

$$
\frac{|\bar{w}(G)|}{q^{(n-1) / 13}}>\frac{|G|}{\left(\frac{q^{n-1}-1}{2}\right)^{1 / 3}}
$$

if $n$ is large enough. This is achieved below where we prove that $|\bar{w}(G)| /|G| \geq$ $\gamma n^{-3} q^{-50-n / 4}$ for some constant $\gamma>0$ depending only on $\bar{w}$.

Let $G=L(q)$ be a quasisimple group of Lie rank $r$ defined over $\mathbb{F}_{q}$. A regular semisimple (r.s.) element $g$ of $G$ is one which has distinct eigenvalues (possibly in a field extension of $\left.\mathbb{F}_{q}\right)$. Therefore $C_{G}(g)$ is a torus of $G$ and so $\left|C_{G}(g)\right|=(1+o(1)) q^{r}$. It is well-known that the set of regular semisimple elements of a semisimple connected algebraic group $G$ has complement of strictly smaller dimension than $\operatorname{dim} G$ (see [GL2]). Using this we have

Lemma 1.3. Given the type $L$ there is a constant $C$ depending on $L$ such that the cardinality of the r.s. elements of $G$ is at least $(1-C / q)|G|$.

The following proposition is an important ingredient for our proof of Theorem 3

Proposition 1.4. Given $\bar{w}=\left\{w_{1}, \ldots, w_{k}\right\}$ there is a constant $c>0$ depending only on $\bar{w}$ such that if $G=S L(4 n, q)$, then $|\bar{w}(G)|>c|G| /\left(n^{3} q^{n-1}\right)$. In fact, $\bar{w}(G)$ contains at least $c q^{3 n} / n^{3}$ conjugacy classes of regular semisimple elements.

Proof. The main idea of the proof is a generalization of some arguments in Section 2 of [LSh1] from the case of $\operatorname{SL}\left(2, q^{n}\right)$ to $\operatorname{SL}\left(4, q^{n}\right)$ :

Consider the inclusion $i: H=S L\left(4, q^{n}\right) \rightarrow S L(4 n, q)$ and let $g \in H$ be a semisimple element whose eigenvalues form the multiset $\left\{a_{1}, \ldots, a_{4}\right\}=A$. Let $F$ be the automorphism $x \mapsto x^{q}$ of $\overline{\mathbb{F}}_{q}$. Since the eigenvalues $a_{i}$ are roots of the characteristic polynomial of $g$ with coefficients in $\mathbb{F}_{q^{n}}$ it follows that $A^{F^{n}}$ is a permutation of $A$. The eigenvalues of $i(g)$ form the multiset $A, A^{F}, \ldots A^{F^{n-1}}$.

Lemma 1.5. There are at least $\left(1-O\left(n q^{-n / 2}\right)\right)|H|$ elements of $g \in H$ such that $i(g)$ is regular semisimple in $G$.

Proof. By Lemma 1.3 the number of elements of $H$ which are not regular semisimple in $H$ is $O\left(q^{-n}\right)|H|$. So it is enough to consider only regular semisimple elements $g \in H$.

Now, suppose that $g \in H$ is regular semisimple but $i(g) \in G$ is not regular. The eigenvalue multiset of $g \in H$ then consists of four distinct elements and has the form

$$
A=\bigcup_{i=1}^{s}\left\{\alpha_{i}, \alpha_{i}^{F^{n}}, \ldots, \alpha_{i}^{F^{\left(k_{i}-1\right) n}}\right\},
$$


where $4=k_{1}+\cdots+k_{s}$ is a partition of 4 , and $\alpha_{i}$ is a generator for the finite field $\mathbb{F} q^{k_{i} n}$ over $\mathbb{F}_{q^{n}}$. In addition, the product of all elements of $A$ should be 1 .

Since $i(q)$ is not regular there are two elements $\alpha$ and $\beta$ from $A$ such that $\alpha=\beta^{F^{j}}$ where $0<j<n$. Now $\alpha$ and $\beta$ are either from distinct orbits of $F^{n}$ in $A$, or from the same orbit. In the first case without loss of generality we may assume that $\alpha=\alpha_{1}$ and $\beta=\alpha_{2}=\alpha_{1}^{j}$. Then $k_{1}=k_{2}$ and the eigenvalue multiset $A$ of $g$ is then determined by $j$ and the $(s-1)$ eigenvalues $\alpha_{i} \in \mathbb{F}_{q^{k_{i}}}$ for $i \neq 2$. Simple calculation shows that the number of possibilities for $A$ (i.e. for the conjugacy class of $g$ in $H=S L\left(4, q^{n}\right)$ ) is at most $O\left(n q^{2 n}\right)$.

In the second case, if we assume that $\alpha=\alpha_{1}$ there is a proper divisor $d$ of $k_{i} n$ such that $\alpha_{1}=\alpha_{1}^{F^{d}}$, i.e. $\alpha_{1} \in \mathbb{F}_{q^{d}}$. Given $d$, counting the possibilities for $\alpha_{1}, \ldots, \alpha_{s}$ under the restriction $\alpha_{1} \in \mathbb{F}_{q^{d}}$ we see that there are $O\left(q^{3 n-k_{i} n+d}\right)=O\left(q^{3 n-n / 2}\right)$ possible choices for $A$. So in this case, as $d$ ranges over all proper divisors of $n$ there are altogether at most $O\left(n q^{5 n / 2}\right)$ conjugacy classes of such $g$.

Combining both cases for all partitions $\left(k_{i}\right)$ of 4 we see that the number of conjugacy classes of regular semisimple elements $g \in H$ such that $i(g)$ is not regular in $\operatorname{SL}(4 n, q)$ is $O\left(n q^{3 n-n / 2}\right)$. This gives the conclusion of the lemma.

Now by Proposition 8.2 of [LSh1] there is a constant $c_{0}>0$ such that $|\bar{w}(H)|>c_{0}|H|$. Together with Lemma 1.5 this implies that $\bar{w}(H)$ contains at least $c_{1} q^{3 n}$ conjugacy classes of r.s. elements $g$ such that $i(g)$ is also regular. Here the constant $c_{1}$ can be taken to be any number in $\left(0, c_{0}\right)$ provided that $n$ is sufficiently large.

Lemma 1.6. Suppose $g^{H}$ is a conjugacy class of r.s. elements of H. There are at most $O\left(n^{3}\right)$ distinct conjugacy classes $h^{H}$ of $H$ such that $i(g)^{G}=i(h)^{G}$.

Proof. Note that the conjugacy classes of semisimple elements of $\operatorname{SL}(n, q)$ are uniquely determined by the multisets of their eigenvalues. Given $g$ with eigenvalue multiset $A$ as above, suppose that $h$ has an eigenvalue multiset $B=\left\{b_{1}, \ldots, b_{4}\right\}$ and $i(g)^{G}=i(h)^{G}$, i.e., $\bigcup_{j=1}^{n-1} A^{F^{j}}=\bigcup_{j=1}^{n-1} B^{F^{j}}$. This implies that every one of $b_{1}, b_{2}, b_{3}$ can take one of $4 n$ given values. Having chosen these three, the fourth one, $b_{4}$, is then uniquely determined by $\operatorname{det} h=1$.

From the above lemma we deduce that $i(\bar{w}(H))$ contains at least $c q^{3 n} / n^{3}$ distinct conjugacy classes of r.s. elements of $G$ (where $c$ depends only on $\bar{w}$ ) and so

$$
|\bar{w}(G)| \geq\left|i(\bar{w}(H))^{G}\right| \geq \frac{c q^{3 n}}{n^{3}} \cdot \frac{|G|}{q^{4 n-1}}=\frac{c|G|}{n^{3} q^{n-1}},
$$

proving Proposition 1.4 .

For general $\operatorname{SL}(n, q)$ we have

Proposition 1.7. Given $\bar{w}$ there is a constant $c^{\prime}>0$ depending only on $\bar{w}$ and such that if $G=S L(n, q)$ then

$$
|\bar{w}(G)|>\frac{c^{\prime}|G|}{n^{3} q^{24+n / 4}}
$$


Proof. Let $m=[n / 4]$. Consider the embedding $j: S L(4 m, q) \rightarrow S L(n, q)$ in the top left corner. By Proposition $1.4, \bar{w}(H)$ contains $c q^{3 m} / m^{3}$ conjugacy classes of r.s. semisimple elements $g$. Observe that this means that $4 m$ of the eigenvalues of $j(g) \in G$ are distinct, and the rest are equal to 1 . This easily gives that $\left|C_{G}(j(g))\right|=O\left(q^{25+4 m-1}\right)$ and hence $|\bar{w}(G)|>c^{\prime}|G| /\left(n^{3} q^{m+24}\right)$ for an appropriate $c^{\prime}$.

\section{The unitary group}

When $L=S U(d, q)$ the result we use is

Proposition 1.8. There is a constant $e>0$ such that

$$
|\bar{w}(L)|>\frac{e|L|}{d^{3} q^{49+d / 4}} .
$$

Proof. The argument is similar to the case of $\operatorname{SL}(n, q)$ above. Let $n \in \mathbb{N}$ be odd. Consider the embedding $i$ of $H=S U\left(4, q^{n}\right)$ in $G=S U(4 n, q)$ defined in the following way: Let $V$ be a 4-dimensional vector space over $\mathbb{F}_{q^{2 n}}$ equipped with a nondegenerate hermitian form $v: V \times V \rightarrow \mathbb{F}_{q^{2 n}}$. Consider the form $v^{\prime}=t \circ v$ where $t=\operatorname{Tr}_{\mathbb{F}_{q^{2 n}} / \mathbb{F}_{q^{2}}}$ is the trace map onto $\mathbb{F}_{q^{2}}$. Since $n$ is odd the above map is still hermitian (and nondegenerate). For $g \in H, i(g)$ is the same transformation $g$ of $V$ considered as a vector space over $\mathbb{F}_{q^{2}}$ with the form $v^{\prime}$.

Let $F$ be the automorphism $x \mapsto x^{q}$. If $g \in H$ is a semisimple element its multiset of four eigenvalues $A$ satisfies $A=A^{-F^{n}}$. The element $i(g) \in G$ has eigenvalue multiset $A, A^{F^{2}}, \ldots, A^{F^{2(n-1)}}$ and from then on the argument is very much the same: First we prove just as in Lemma 1.5 that the number of elements $g \in H$ such that $i(g)$ is not r.s. is $O\left(n q^{-n / 2}|H|\right)$. Then, just as in Proposition 1.4 it follows that there is a constant $e_{0}$ such that at least $e_{0} q^{3 n}$ conjugacy classes of $H$ consist of elements $g \in \bar{w}(H)$ such that $i(g)$ is regular semisimple. Next, at most $O\left(n^{3}\right)$ such conjugacy classes of $H$ become conjugate in $G$ (because for $i(g)^{G}=i(h)^{G}$ we need three eigenvalues of $h$ to be from $A^{ \pm F^{2 j}}, j=0,1, \ldots, n-1$, and then they determine the last eigenvalue of $\left.h\right)$. It follows that $\bar{w}(G)$ contains at least $e_{1} q^{3 n} / n^{2}$ conjugacy classes of r.s. elements. Finally, considering the embedding of $G=S U(4 n, q)$ in $L=S U(d, q)$ as a subspace group (where $n=2[(d-4) / 8]+1$ ), we see that $\bar{w}(L)$ contains at least $e_{1} q^{3 n} / n^{2}$ conjugacy classes of elements $g$ with distinct eigenvalues on a nondegenerate $4 n$-dimensional subspace $U$. Any $h \in C_{L}(g)$ stabilizes $U$ and $U^{\perp}$ and hence $\left|C_{L}(g)\right|=O\left(q^{4 n+49}\right)$. (Note that $\operatorname{dim} U^{\perp}=d-4 n \leq 7$.)

This completes the proof of Theorem 3 . Incidentally we observe the following consequence of Propositions 1.2, 1.7 and 1.8 .

Theorem 1.9. Given a set of words $\bar{w}$ and a simple group $L$ of Lie type, two random elements from $\bar{w}(L)$ generate $L$ with probability tending to 1 as $|L| \rightarrow \infty$. In particular a random pair of squares generates $L$ with probability 1.

This follows easily from the above propositions and a result of Liebeck and Shalev in [LSh3] that the set of pairs $(a, b) \in L \times L$ which do not generate $L$ has size at most $c|L|^{2} / P(L)<c^{\prime}|L|^{2} q^{-r}$ for some constants $c, c^{\prime}$. Here and below $P(G)$ denotes the 
minimal degree of a faithful permutation representation of a group $G$. When $G=L$ is simple this is the same as the minimal index of a proper subgroup of $L$.

It was recently proved in [Sh2] that an analogous result holds for alternating groups.

\section{Bounds for linear groups}

By a classical result of Jordan a finite linear group of degree $k$ has an abelian normal subgroup $A$ of index $j(k)$ for some function $j(k)$. Essentially the best elementary estimate for $j(k)$ is due to Blichfeldt:

$$
j(k) \leq k ! 6^{(k-1) \pi(k+1)+1}
$$

where $\pi(k+1)$ denotes the number of primes $\leq k+1$ (see [Do]).

Better bounds can be obtained using CFSG. Building on an unpublished work of Weisfeiler, Collins [Co] has recently shown that for $k \geq 71$ we have $j(k)=(k+1)$ ! (see [GL1] for the history of this result).

It is clear that $G / A$ has an embedding into $\operatorname{Sym}(j(k))$. Hence Theorem 4 may be considered as a different type of quantitative version of Jordan's theorem.

For the proof we need various auxiliary results.

A $p$-group $P$ is said to be of symplectic type if it has no noncyclic characteristic abelian subgroups. The structure of these groups is well understood and is closely related to that of extraspecial groups.

In particular if $P$ has exponent $p$ ( $p$ odd), then $P$ itself is extraspecial, and if it has exponent 4 , then $P$ is either extraspecial or the central product of an extraspecial group and $\mathbb{Z}_{4}$.

We will use the following.

Proposition 2.1. Let $P$ be a p-group of symplectic type and set $C=C_{\operatorname{Aut}(P)}(Z(P))$.

(i) If $P$ has exponent $p$ (with $p$ odd) and order $p^{2 m+1}$, then $C$ can be embedded in $\operatorname{Sym}\left(p^{2 m}\right)$.

(ii) If $P$ has exponent 4 and order $2^{2 m+2}$, then $C$ can be embedded in $\operatorname{Sym}\left(2^{2 m+2}-4\right)$.

(iii) If $P$ has exponent 4 and order $2^{2 m+1}$, then $C$ can be embedded in $\operatorname{Sym}\left(2^{2 m+1}-2\right)$.

Hence in all cases $C$ can be embedded in $\operatorname{Sym}\left(4 p^{2 m}\right)$.

Proof. The structure of the above groups $P$ and $C$ is described in [KL, Table 4.6A]. In all cases $\operatorname{Inn}(P)$ is an elementary abelian minimal normal subgroup of order $p^{2 m}$ in $C$. Each element of $C / \operatorname{Inn}(P)$ acts as a nontrivial linear transformation, hence $\operatorname{Inn}(P)$ is the unique minimal normal subgroup of $C$.

In case (i) we have $C / \operatorname{Inn}(P) \cong \operatorname{Sp}(2 m, p)$ and moreover by [Gr] the extension splits. Hence $C$ has a corefree subgroup of index $p^{2 m}$, which implies (i).

In case (ii) $P$ may be expressed as the central product of $\mathbb{Z}_{4}$ and $m$ copies of the dihedral group $D_{8}, P=\mathbb{Z}_{4} \circ D_{8} \circ \cdots \circ D_{8}$.

In case (iii) either $P$ is a central product of $m$ copies of $D_{8}$, or $P$ is a central product of $m-1$ copies of $D_{8}$ with the quaternion group $Q_{8}$. 
In both cases $C$ acting faithfully on the elements of $P \backslash Z(P)$ provides the required embedding.

For a finite group $G$ we denote by $R_{0}(G)$ the smallest degree of a nontrivial complex projective representation of $G$. For simple groups $L$ of Lie type strong lower bounds for $R_{0}(L)$ follow from the work of Landazuri and Seitz [LS] (see [KL, Table 3.3A] and [Lü]). See also [KL] and [CCNPW] for the value of $R_{0}(L)$ when $L$ is an alternating or sporadic group.

Recall that $P(G)$ denotes the minimal degree of a faithful permutation representation of a group $G$. If $L$ is a simple group with a proper subgroup $H$, then $H$ considered as a subgroup of $\operatorname{Aut}(L)$ is corefree, hence we have

$$
P(\operatorname{Aut}(L)) \leq|L: H||\operatorname{Out}(L)| .
$$

When $L$ is of Lie type and $H$ is a parabolic subgroup of $L$ we can improve the above bound as follows: The group $H$ is invariant under the subgroups $D, \Phi \leq \operatorname{Aut}(L)$ of diagonal and field automorphisms of $L$. Then $H D \Phi \leq \operatorname{Aut}(L)$ is a corefree subgroup and hence we have

$$
P(\operatorname{Aut}(L)) \leq 6|L: H|
$$

if $H$ is parabolic. Sharp bounds for $P(L)$ when $L$ is a classical simple group can be found in [KL, Table 5.2A] and they are achieved for parabolic subgroups.

If $L$ is an exceptional group of Lie type one can easily find a maximal parabolic subgroup $P$ of small index. A good lower bound for the order of $P$ follows by noting that $|P|$ is divisible by the order of a Borel subgroup of $G$ and also by the order of the Levi factor corresponding to $P$ (see [KL, pp. 179-181] for a quick account of these standard facts). See also [Wi] for the detailed structure of many of these maximal subgroups.

Small index subgroups in sporadic simple groups can be found in [CCNPW].

In the proof of Theorem 4 we use CFSG via the following.

Proposition 2.2. There is an absolute constant $c_{0}$ such that if $L$ is a nonabelian finite simple group then

$$
P(\operatorname{Aut}(L)) \leq c_{0} R_{0}(L)^{2} .
$$

In fact $c_{0}$ can be taken to be $10^{10}$.

Proof. This follows easily from the above mentioned results by inspection.

Note that the bound with $c_{0}=10^{10}$ is quite sharp for the Monster simple group.

Recall that a group $H$ is quasisimple if it is a perfect, central extension of a simple group $L$. It is known [Hu] that $\operatorname{Aut}(H)$ is isomorphic to a subgroup of $\operatorname{Aut}(L)$.

The components of a group are its subnormal quasisimple subgroups. The subgroup $E=E(G)$ is generated by the components of $G$. The components of $E(G)$ are exactly the components $C_{j}$ of $G$ and $\operatorname{Aut}(E)$ permutes the components among themselves [As].

Denote the orbits of $\operatorname{Aut}(E)$ on the components by $B_{k}(k=1,2, \ldots)$. Then $B_{k}$ consists of $t_{k}$ isomorphic components with central quotient $L_{k}$. The automorphism group of $B_{k}$ has a natural embedding into $\operatorname{Aut}\left(L_{k}\right)$ i $\operatorname{Sym}\left(t_{k}\right)$. Moreover $\operatorname{Aut}(E)$ has an embedding into $\prod_{k} \operatorname{Aut}\left(B_{k}\right)$. These observations imply the following 


\section{Proposition 2.3.}

$$
P(\operatorname{Aut}(E)) \leq \sum_{j} P\left(\operatorname{Aut}\left(C_{j} / Z\left(C_{j}\right)\right)\right)
$$

where the sum is taken over all components $C_{j}$ of $G$.

The generalised Fitting subgroup of $G$ is $F^{*}(G)=E(G) F(G)$ (where $F(G)$ is the Fitting subgroup of $G)$. The most significant fact about $F^{*}(G)$ is that $C_{G}\left(F^{*}(G)\right) \leq F^{*}(G)$. Denote $C_{G}\left(F^{*}(G)\right)=Z\left(F^{*}(G)\right)$ by $Z$.

The subgroups $E$ and the Sylow subgroups $O_{p}(G)$ of $F(G)$ are characteristic in $G$ and their product is $F^{*}(G)$. Hence $G / Z$ has a natural embedding into

$$
\operatorname{Aut}(E) \times \prod_{p|| F(G) \mid} \operatorname{Aut}\left(O_{p}(G)\right) .
$$

Recall that an irreducible linear group $G \leq G L(V)$ is called imprimitive if the vector space $V$ can be decomposed into a direct sum $V=V_{1} \oplus \cdots \oplus V_{t}$ with $t>1$ such that every element of $G$ permutes the subspaces $V_{i}$ among themselves, and $G$ is primitive if no such decomposition exists. By Clifford's theorem any normal subgroup $N$ of a primitive group is homogeneous, in particular $N$ acts faithfully and irreducibly on some subspace $W$ such that $\operatorname{dim} W \operatorname{divides} \operatorname{dim} V$. For primitive linear groups we prove the following more precise version of Theorem 4

Theorem 2.4. Let $G$ be a finite primitive subgroup of $G L(k, \mathbb{C})$. Then $G / Z(G)$ has an embedding into $\operatorname{Sym}\left(c_{0} k^{2}\right)$.

Proof. It is known [Di] that $Z=Z(G)$ is the unique maximal normal abelian subgroup of $G$, hence $Z=Z\left(F^{*}(G)\right)$. Moreover, $Z$ is a group of scalars, hence cyclic.

Let $p$ be a prime such that $O_{p}(G)$ is not contained in $Z$. Then $O_{p}(G)$ is the product of the Sylow $p$-subgroup $Z_{p}$ of $Z$ and an extraspecial $p$-group which is of exponent $p$ in case $p \neq 2$ [LMM, Lemma 1.7]. Therefore the elements of order $p(\operatorname{resp} . \leq 4)$ in $O_{p}(G)$ form a characteristic subgroup $R_{p}$ of $G$ of symplectic type and $O_{p}(G)=Z_{p} \cdot R_{p}$.

By the remarks preceding the theorem $G / Z$ has a natural embedding into $\operatorname{Aut}(E) \times$ $\prod_{p} \operatorname{Aut}\left(O_{p}(G)\right)$. Since conjugation by elements of $G$ stabilizes $R_{p}$ and fixes $Z_{p}$ elementwise we actually have an embedding of $G / Z$ into $\operatorname{Aut}(E) \times \prod_{p} C_{\operatorname{Aut}\left(R_{p}\right)}\left(Z\left(R_{p}\right)\right)$, where the product is taken over all primes $p$ such that $O_{p}(G) \neq Z_{p}$.

Consider the normal subgroup $N=E \cdot \prod_{p} R_{p}$ of $G$. The group $N$ may be considered to be an irreducible subgroup of $G L(W)$ for some $W$ where $\operatorname{dim}(W)$ divides $k . N$ is a central product of the symplectic type groups $R_{p}$ and the components $C_{i}$ of $G[\mathrm{As}]$. Hence there is a decomposition of $W$ into the tensor product of spaces $\left\{W_{p}\right\}$ and $\left\{W_{i}\right\}$ such that $W_{p}$ is an irreducible $R_{p}$-module for all $p$ and $W_{i}$ is an irreducible $C_{i}$-module for all $i$ [Gor, 3.7.1 and 3.7.2].

It is clear that $\operatorname{dim}\left(W_{i}\right) \geq R_{0}\left(C_{i} / Z\left(C_{i}\right)\right)$. We have

$$
k \geq \prod_{p} \operatorname{dim}\left(W_{p}\right) \cdot \prod_{i} \operatorname{dim}\left(W_{i}\right) .
$$


Moreover, if $R_{p}$ has order $p^{2 m_{p}+1}$ or $p^{2 m_{p}+2}$, then $\operatorname{dim}\left(W_{p}\right)=p^{m_{p}}$ [Gor, 5.5.5]. Hence we have $k \geq \prod_{p} p^{m_{p}} \cdot \prod_{i} R_{0}\left(C_{i} / Z\left(C_{i}\right)\right)$, which gives

$$
\sum_{p} p^{2 m_{p}}+\sum_{i} R_{0}\left(C_{i} / Z\left(C_{i}\right)\right)^{2} \leq k^{2} .
$$

But $G / Z$ has a faithful permutation representation of degree at most

$$
\begin{aligned}
& \sum_{p} P\left(C_{\operatorname{Aut}\left(R_{p}\right)}\left(Z\left(R_{p}\right)\right)\right)+P(\operatorname{Aut}(E)) \\
& \quad \leq c_{0}\left(\sum_{p} p^{2 m_{p}}+\sum_{i} R_{0}\left(C_{i} / Z\left(C_{i}\right)\right)^{2}\right) \leq c_{0} k^{2}
\end{aligned}
$$

using Propositions 2.1 2.2 and 2.3 Our statement follows.

The end of the proof of Theorem 4 . If $G \leq G L(k, \mathbb{C})$ is irreducible but imprimitive, then it can be embedded into a wreath product $G_{1} 2 T$ where $G_{1}$ is a primitive subgroup of $G L\left(k_{0}, \mathbb{C}\right), T$ is a transitive subgroup of $\operatorname{Sym}(t)$ and $t k_{0}=k[\mathrm{Su}]$. Now $A=Z\left(G_{1}\right)^{t}$ is an abelian normal subgroup of $G_{1} 2 T$.

We have $(G A) / A \leq\left(G_{1} 2 T\right) / A$ and by Theorem 2.4, $\left(G_{1} 2 T\right) / A$ has an embedding into $\operatorname{Sym}\left(c_{0} k_{0}^{2} t\right)$. Hence $G / G \cap A$ has an embedding into $\operatorname{Sym}\left(c_{0} k^{2}\right)$.

Finally, if $G \leq G L(k, \mathbb{C})=G L(V)$ (with $V=\mathbb{C}^{k}$ ) is an arbitrary finite linear group then $G$ is completely reducible. Hence it embeds into a direct product $\prod_{j} G L\left(V_{j}\right)$ where $V=\bigoplus_{j} V_{j}$ is a decomposition of $V$ into irreducible $\mathbb{C} G$-modules. Let $k_{j}=\operatorname{dim}_{\mathbb{C}} V_{j}$, so that $\sum_{j} k_{j}=k$. Our group $G$ acts irreducibly on each $V_{j}$ and so by the argument above we find a subgroup $A_{j} \leq G$ such that its image in $G L\left(V_{j}\right)$ is abelian and $G / A_{j}$ embeds in $\operatorname{Sym}\left(c_{0} k_{j}^{2}\right)$. Take $A=\bigcap_{j} A_{j}$. It follows that $A$ is abelian and $G / A$ embeds in $\operatorname{Sym}\left(\sum_{j} c_{0} k_{j}^{2}\right) \leq \operatorname{Sym}\left(c_{0} k^{2}\right)$. Theorem 4 follows.

By a result of Easdown and Praeger $[\mathrm{EP}]$ if $G$ is a subgroup of $\operatorname{Sym}(t)$, then $G / \operatorname{Sol}(G)$ can also be embedded into $\operatorname{Sym}(t)$ (where $\operatorname{Sol}(G)$ is the soluble radical of $G$ ). Hence Theorem 4 has the following immediate consequence.

Corollary 2.5. Let $G$ be a finite linear group of degree $k$. Then $G / \operatorname{Sol}(G)$ has an embedding into $\operatorname{Sym}\left(c_{0} k^{2}\right)$.

In particular $G / \operatorname{Sol}(G)$ is a linear group of degree at most $c_{0} k^{2}$.

Question 2.6. Suppose $G$ is a finite linear group of degree $k$. Is it true that $G / F(G)$ embeds in $\operatorname{Sym}\left(c k^{2}\right)$ for some constant $c$ ? What about $G / \operatorname{Frat}(G)$ ?

\section{Additional remarks}

Following [Gow] let us put some of the results in this paper in a more general context.

If the minimal degree of a representation of a group $G$ is at least 2 , then the group is perfect (i.e. equal to its commutator subgroup). Hence it is reasonable to assume that a quasirandom group is perfect. For diverse examples of such groups see [HP]. 
Theorem 3.1. Let $G$ be a perfect group of order $n$. Then the following statements are polynomially equivalent, in the sense that if one statement holds for a constant $c$, then all others hold with constants that are bounded by a positive power of $c$.

(i) Every nontrivial representation of $G$ has degree at least $c_{1}$.

(ii) Any product-free subset of $G$ has size at most $n / c_{2}$.

(iii) For any subset $B$ of size at least $n / c_{3}$ we have $B^{3}=G$.

(iv) Every proper subgroup of $G$ has index at least $c_{4}$.

(v) Let $m \geq 2$ be an integer. Then for every subset $S$ of $G$ of size at least $n / a_{m}$ (where $a_{m}$ depends on $\left.m\right) S$ contains $m$ elements $x_{1}, \ldots, x_{m}$ together with all subproducts $x_{1}^{\varepsilon_{1}} \ldots x_{m}^{\varepsilon_{m}}$, where $\varepsilon_{1} \in\{0,1\}$ (and at least one of the $\varepsilon_{i}$ is 1 ).

Proof. (i) $\Rightarrow$ (ii) follows from Proposition 0 (due to Gowers).

(i) $\Rightarrow$ (iii) follows from Corollary 1

(iv) is an easy consequence of either (ii) or (iii).

(iv) $\Rightarrow$ (i) follows from Theorem 4

(i) $\Rightarrow$ (v) is a special case of Corollary 5.4 of [Gow].

(ii) is a special case $m=2$ of (v) (indeed it is quite surprising that this case is polynomially equivalent to the cases $m=3,4, \ldots$ etc.)

Finally, let us point out an application of Corollary 1 to permutation groups. By a deep result of Fulman and Guralnick [FG] if $G$ is a simple group acting transitively on a set $X$ then the proportion of fixed-point-free permutations in $G$ is at least $\delta$ for some absolute constant $\delta>0$. This implies that if $G$ is large enough, then each element of $G$ is a product of three fixed-point-free permutations.

\section{Appendix: a short proof of a special case of Proposition 0}

In this section we will give a short version of Gowers' proof of Proposition 0 in the case when one of the sets $A, B, C$ is symmetric. This case is enough for most of the applications above. We stress that we do not claim originality: certainly all the elements of the argument below are already present in Gowers' proof in [Gow]. We believe that it is worth presenting a simplified version which can fit in one page.

Proof. Suppose $A, B, C$ are three subsets of $G$ one of which coincides with its inverse and such that $|A||B||C|>n^{3} / k$. We have to show that the equation $a b=c$ has a solution with $a \in A, b \in B$ and $c \in C$. By cyclically permuting and inverting some of $A, B, C$ we may assume without loss of generality that $B=B^{-1}$ is a symmetric set.

Let $V=\mathbb{C} G$ be the group algebra over $\mathbb{C}$ considered as a complex vector space with basis $G$. We will consider $V$ as a left $G$-module equipped with the standard Hermitian inner product (so the elements $\mathbf{g}$ of $G \subset V$ form an orthonormal basis of $V$ ).

Let $X=\left(x_{g, h}\right)$ be the $n$ by $n$ matrix labelled by $g, h \in G$ such that $x_{g, h}=0$ if $h^{-1} g \notin B$ and $x_{g, h}=1$ otherwise. Then $X$ is a real symmetric matrix defining a linear map $X \in$ End $_{\mathbb{C}} V$. For each $\mathbf{u} \in G \subset V$ we have $X \mathbf{u}=\sum_{b \in B} \mathbf{u b}$, which shows that $X$ is in fact a $G$-module endomorphism, i.e. $X g \mathbf{v}=g X \mathbf{v}$ for all $g \in G$ and $\mathbf{v} \in V$. 
Note that every row and column sum of $X$ is $|B|$, hence $\lambda_{1}=|B|$ is an eigenvalue of $X$ with eigenvector $\mathbf{e}=\sum_{g \in G} \mathbf{g} \in V$. Let $I=\mathbf{e}^{\perp}$ be the augmentation ideal of $V$. It is both $X$ - and $G$-invariant and clearly does not have $G$-invariant vectors.

Now, since $X$ is symmetric it has real eigenvalues. Let $\lambda \in \mathbb{R}$ be an eigenvalue of $X$ on $I$ with eigenspace $V_{\lambda}$. Since $X$ is a $G$-endomorphism it follows that $V_{\lambda}$ is a nontrivial $G$-module and hence $\operatorname{dim} V_{\lambda} \geq k$.

The eigenvalues of $X^{2}$ are exactly the squares of the eigenvalues of $X$ with the same multiplicities. Thus we have $\operatorname{tr}\left(X^{2}\right) \geq k \lambda^{2}$. But $\operatorname{tr}\left(X^{2}\right)=\operatorname{tr}\left(X^{t} X\right)$ is the sum of all entries of $X$, which is $n|B|$. It follows that $\lambda^{2} \leq n|B| / k$ for all eigenvalues $\lambda$ of $X$ on $I$ and therefore

$$
|X \mathbf{v}|^{2} \leq \frac{n|B|}{k}|\mathbf{v}|^{2} \quad \text { for all } \mathbf{v} \in I
$$

Let $\mathbf{v}=n \sum_{g \in A} \mathbf{g} \in V$. We can write $\mathbf{v}=\mathbf{v}_{1}+\mathbf{v}_{2}$ where $\mathbf{v}_{1}=|A| \mathbf{e}$ and $\mathbf{v}_{2} \in I$ with $\left|\mathbf{v}_{2}\right|^{2}=|A| n(n-|A|)<n^{2}|A|$. Assuming that $a b=c$ has no solution $a \in A, b \in B$, $c \in C$ we deduce from the definition of $X$ that $X \mathbf{v} \in \sum_{g \in G \backslash C} \mathbb{C g}$. However

$$
X \mathbf{v}=X \mathbf{v}_{1}+X \mathbf{v}_{2}=|B||A| \mathbf{e}+X \mathbf{v}_{2}
$$

and it follows that the vector $X \mathbf{v}_{2}$ has coordinates equal to $-|B||A|$ in at least $|C|$ positions, so $\left|X \mathbf{v}_{2}\right|^{2} \geq|C||A|^{2}|B|^{2}$. On the other hand, by 1 ,

$$
|C||A|^{2}|B|^{2} \leq\left|X \mathbf{v}_{2}\right|^{2} \leq \frac{n|B|}{k}\left|\mathbf{v}_{2}\right|^{2} \leq \frac{n|B|}{k} n^{2}|A|,
$$

which implies $|A||B||C| \leq n^{3} / k$ and this contradicts the starting assumption. Hence $a b=c$ has a solution as stated in the proposition.

Acknowledgments. We would like to thank Vera T. Sós for drawing our attention to the paper of Gowers, Aner Shalev for sending us his yet unpublished paper [Sh1] and Balázs Szegedy for some enlightening discussions. This research was supported in part by OTKA grants NK 72523 and NK 78439 .

\section{References}

[As] Aschbacher, M.: Finite Group Theory. Cambridge Univ. Press (1986) Zbl 0583.20001 MR 0895134

[Ba] Babai, L.: On the diameter of Eulerian orientations of a graph. In: Proc. 17th Ann. Sympos. Discrete Algorithms (SODA’06), ACM-SIAM, 822-831 (2006) Zbl 1192.05084 MR 2368881

[BS] Babai, L., Sós, V. T.: Sidon sets in groups and induced subgraphs of Cayley graphs. Eur. J. Combin. 6, 101-114 (1985) Zbl 0573.05032 MR 0810691

[Co] Collins, M.: On Jordan's theorem for complex linear groups. J. Group Theory 10, 411423 (2007) Zbl 1125.20033 MR 2334748

[CCNPW] Conway, J. H., Curtis, R. T., Norton, S. P., Parker, R. A., Wilson, R. A.: An Atlas of Finite Groups. Oxford Univ. Press (1985) Zbl 0568.20001 MR 0827219

[Di] Dixon, J. D.: The Structure of Linear Groups. Van Nostrand-Reinhold, Princeton (1971) Zbl 0232.20079 MR 0347959 
[Do] Dornhoff, L.: Group Representation Theory. Part A: Ordinary Representation Theory. Pure Appl. Math. 7, Dekker, New York (1971) Zbl 0227.20002

[EP] Easdown, D., Praeger, C. E.: On minimal faithful permutation representations of finite groups. Bull. Austral. Math. Soc. 38, 207-220 (1988) Zbl 0661.20012 MR 0969911

[FG] Fulman, J., Guralnick, R. M.: Derangements in simple and primitive groups. In: Groups, Combinatorics and Geometry (Durham 2001), World Sci., 99-121 (2003) Zbl 1036.20002 MR 1994962

[Gor] Gorenstein, D.: Finite Groups. Harper and Row, New York (1968) Zbl 0185.05701 MR 0231903

[Gow] Gowers, W. T.: Quasirandom groups. Combin. Probab. Comput. 17, 363-387 (2008) Zbl 1191.20016 MR 2410393

[GR] Green, B., Ruzsa, I. Z.: Sum-free sets in abelian groups. Israel J. Math. 147, 157-188 (2005) Zbl 1158.11311 MR 2166359

[Gr] Griess, R. L., Jr.: Automorphisms of extra special groups and nonvanishing degree 2 cohomology. Pacific J. Math. 48, 403-422 (1973) Zbl 0283.20028 MR 0476878

[GL1] Guralnick, R. M., Lorenz, M.: Orders of finite groups of matrices. In: Groups, Rings and Algebras, Contemp. Math. 420, Amer. Math. Soc., Providence, RI, 141-161 (2006) Zbl 1203.20042 MR 2279238

[GL2] Guralnick, R. M., Lübeck, F.: On $p$-singular elements in Chevalley groups in characteristic $p$. In: Groups and Computation. III (Columbus, OH, 1999), Ohio State Univ. Math. Res. Inst. Publ. 8, de Gruyter, Berlin, 169-182 (2001) Zbl 1001.20045 MR 1829478

[He1] Helfgott, H. A.: Growth and generation in $S L_{2}(\mathbb{Z} / p \mathbb{Z})$. Ann. of Math. 167, 601-623 (2008) Zbl pre05578700 MR 2415382

[He2] Helfgott, H. A.: Growth in $S L_{3}(\mathbb{Z} / p \mathbb{Z})$. J. Eur. Math. Soc. 13, 761-851 (2011) Zbl pre05873844

[HP] Holt, D. F., Plesken, W.: Perfect Groups. Oxford Univ. Press (1989) Zbl 0691.20001 MR 1025760

[Hu] Hughes, N. J. S.: The structure and order of the group of central automorphisms of a finite group. Proc. London Math. Soc. 52, 377-385 (1951) Zbl 0042.02301 MR 0041129

[Ke] Kedlaya, K. S.: Product-free subsets of groups. Amer. Math. Monthly 105, 900-906 (1998) Zbl 0986.20025 MR 1656927

[KL] Kleidman, P. B., Liebeck, M. W.: The Subgroup Structure of the Finite Classical Groups. London Math. Soc. Lecture Note Ser. 129, Cambridge Univ. Press (1990) Zbl 0697.20004 MR 1057341

[LS] Landazuri, V., Seitz, G. M.: On the minimal degrees of projective representations of the finite Chevalley groups. J. Algebra 32, 418-443 (1974) Zbl 0325.20008 MR 0360852

[La] Larsen, M.: Word maps have large image. Israel J. Math. 139, 149-156 (2004) Zbl 1130.20310 MR 2041227

[LSh1] Larsen, M., Shalev, A.: Word maps and Waring type problems. J. Amer. Math. Soc. 22, 437-466 (2009) Zbl 1206.20014 MR 2476780

[LSh2] Liebeck, M. W., Shalev, A.: Fuchsian groups, finite simple groups, and representation varieties. Invent. Math. 159, 317-367 (2005) Zbl 1134.20059 MR 2116277

[LSh3] Liebeck, M. W., Shalev, A.: Simple groups, probabilistic methods, and a conjecture of Kantor and Lubotzky. J. Algebra 184, 31-57 (1996) Zbl 0870.20014 MR 1402569

[Lü] Lübeck, F.: Smallest degrees of representations of exceptional groups of Lie type, Comm. Algebra 29, 2147-2169 (2001) Zbl 1004.20003 MR 1837968 
[LMM] Lucchini, A., Menegazzo, F., Morigi, M.: On the number of generators and composition length of finite linear groups. J. Algebra 243, 427-447 (2001) Zbl 0992.20033 MR 1850640

[Sh1] Shalev, A.: Word maps, conjugacy classes, and a non-commutative Waring-type theorem. Ann. of Math. 170, 1383-1416 (2009) Zbl 1203.20013 MR 2600876

[Sh2] Shalev, A.: Mixing and generation in simple groups. J. Algebra 319, 3075-3086 (2008) Zbl 1146.20057 MR 2397424

[S] Street, A. P.: Sum-free sets. In: Lecture Notes in Math. 292, Springer, 123-272 (1972) MR 0392580

[Su] Suprunenko, D. A.: Matrix Groups. Amer. Math. Soc., Providence (1976) Zbl 0317.20028 MR 0390025

[Wi] Wilson, R. A.: Finite Simple Groups. Book in preparation 